\title{
Prostate-specific antigen bounce predicts for a favorable prognosis following brachytherapy: a meta-analysis
}

\author{
Michael B. Bernstein, MD', Nitin Ohri, MD², James W. Hodge, PhD³, Madhur Garg, MD', William Bodner, MD! \\ Shalom Kalnicki, MD', Adam P. Dicker, MD, PhD², Chandan Guha, MD, PhD' \\ 'Department of Radiation Oncology, Albert Einstein College of Medicine/Montefiore Medical Center, Bronx, NY, ${ }^{2}$ Department of Radiation \\ Oncology, Kimmel Cancer Center, Jefferson Medical College of Thomas Jefferson University, Philadelphia, PA, ${ }^{3}$ Laboratory of Tumor \\ Immunology and Biology. Center for Cancer Research, National Cancer Institute, National Institutes of Health, Bethesda, MD, USA
}

\begin{abstract}
Purpose: Controversy exists whether the prostate-specific antigen (PSA) bounce phenomenon following definitive radiation for prostate cancer has prognostic significance. Here, we perform a meta-analysis to determine the association between PSA bounce and biochemical control after brachytherapy alone.

Material and methods: We reviewed Medline, EMBASE, and CENTRAL citations through February 2012. Studies that recorded biochemical failure rates in bouncers and non-bouncers were included. Hazard ratios describing the impact of bounce on biochemical failure were extracted directly from the studies or calculated from survival curves. Pooled estimates were obtained using the inverse variance method. A random effects model was used in cases of significant effect heterogeneity ( $p<0.10$ using $Q$ test).

Results: The final analysis included 3011 patients over 6 studies treated with brachytherapy. Meta-analysis revealed that patients experiencing PSA bounce after brachytherapy, conferred a decreased risk of biochemical failure (random effects model HR $=0.42,95 \%$ CI: $0.30-0.59 ; p<0.001$ ).

Conclusions: Our meta-analysis determined that PSA bounce predicts for improved biochemical control following brachytherapy. To our knowledge, this is the first study describing this effect.

Key words: brachytherapy, prostate cancer, PSA bounce.

\section{Purpose}

The National Comprehensive Cancer Network guidelines recommend prostate-specific antigen (PSA) testing every six to twelve months after definitive radiation therapy for prostate cancer to assess a patient's disease status. Following treatment with brachytherapy, decline of PSA levels to an eventual nadir may take up to five years [1]. In up to $30-40 \%$ patients receiving prostate brachytherapy $[2,3]$, PSA levels may fluctuate without any apparent signs of disease recurrence or progression, which is referred to as a PSA bounce. While PSA bounce is a well-known physiologic phenomenon, its etiology remains unclear. Certain studies have reported that age [3-7], prostate volume and dosimetry $[1,6,8]$, and pre-treatment PSA [9] may predict for a post-radiation PSA bounce. Other precipitating factors may include ejaculations, instrumentations, or prostatitis [10].

While the potential causes of a PSA bounce are continuously being investigated, the clinical significance of a PSA bounce remains highly controversial. Certain studies have failed to show an association between the presence of a bounce and biochemical failure [3,9]. Conversely, a recent study found the presence of a PSA bounce after prostate brachytherapy is strongly related to better outcomes not only in terms of biochemical failure, but also translated to an overall survival benefit [11]. Because of considerable disagreement regarding the prognostic significance of a PSA bounce and the consequent concern that a rising PSA result engenders in patients and treating physicians, we performed an up-to-date meta-analysis of published reports to determine whether any correlation between PSA bounce and biochemical failure exists after definitive brachytherapy alone in prostate cancer patients.
\end{abstract}

Address for correspondence: Chandan Guha, MD, PhD, Professor and Vice Chair, Department of Radiation Oncology, Department of Pathology, Mazer Building, Room-620, Montefiore Medical Centre, Albert Einstein College of Medicine, 1300 Morris Park Avenue, Bronx, New York 10461, USA,

phone: +1 718-920-4321, e-mail: cguha@montefiore.org
Received: 22.08.2013

Accepted: 17.10.2013

Published: 31.12.2013 


\section{Material and methods}

\section{Selection of studies}

We reviewed relevant Medline, EMBASE, and the Cochrane Central Register of Controlled Trials (CENTRAL) citations through February 2012. The search terms used were "PSA bounce" and "PSA bouncing", and returned results were limited to reports published in English. Studies that included patients with non-metastatic prostate cancer, and reported rates of biochemical failure in patients with and without PSA bounces after brachytherapy alone were analyzed. Reviews, supplements, and case reports were excluded. Studies that utilized radiotherapy techniques other than brachytherapy (i.e. external beam radiotherapy [EBRT], stereotactic body radiation therapy or high-intensity focused ultrasound) or combined modality therapy were also excluded. Of note, in one study that was included in the brachytherapy analysis, 4 patients also received EBRT as part of their therapy [8]. However, this study was still included in our final data set as these patients only represented $0.001 \%$ of the study population. When more than one publication was identified from the same study population, the most recent data were used in the final analysis.

\section{Data extraction}

Data extraction was conducted independently by two investigators (M.B.B. and N.O.) according to the Preferred Reporting Items for Systematic Reviews and Meta-analyses (PRISMA) statement [12]. We recorded the first author's last name, journal name and year of publication, inclusion criteria, number of patients, and median age, pre-treatment characteristics, median follow-up, treatment modality, and dose, androgen deprivation therapy (ADT) use, definitions of PSA bounce and biochemical failure, bounce rates, peak bounce values, and median time to first bounce. When multiple definitions for PSA bounce and biochemical recurrence were presented in a single publication, the most commonly used definitions in the literature (i.e. a rise of $0.2 \mathrm{ng} / \mathrm{mL}$ and the ASTRO-Phoenix definition) were chosen whenever possible.

Hazard ratios (HRs) for biochemical failure associated with PSA bounce were extracted directly from the original studies or were estimated indirectly by reading off survival curves as suggested by Parmar et al. [13]. Data extraction from survival curves and estimations of effect sizes were performed using customized scripts in Matlab (The Mathworks, Natick, MA, U.S.A.).

\section{Statistical analysis}

Meta-analysis was performed using the inverse variance method. The Cochran Q statistic was used to assess statistical heterogeneity in effect sizes across trials. The assumption of homogeneity was considered invalid for $p$-values less than 0.10 . This prompted the use of the random-effects model to derive summary estimates for HRs and $95 \%$ CIs. Publication bias was evaluated visually with funnel plots and statistically using the Egger test [14]. A two-tailed $p$-value of less than 0.10 was considered statistically significant.

\section{Results}

\section{Selection of studies}

Using the aforementioned search terms, a total of 115 studies (44 from Medline, 71 from EMBASE, and 0 from Cochrane Central Register of Controlled Trials) were initially returned. After excluding articles for not meeting the criteria detailed in Material and methods, 23 studies were read carefully in their entirety. As shown in Figure 1, 17 studies were excluded either for not recording biochemical failure rates in patients with and without PSA bounce or because of a more recent publication reporting on the same study population. Ultimately, six brachytherapy studies $[1,5-8,11]$ were included in the final analysis. The characteristics of each study are shown in Table 1.

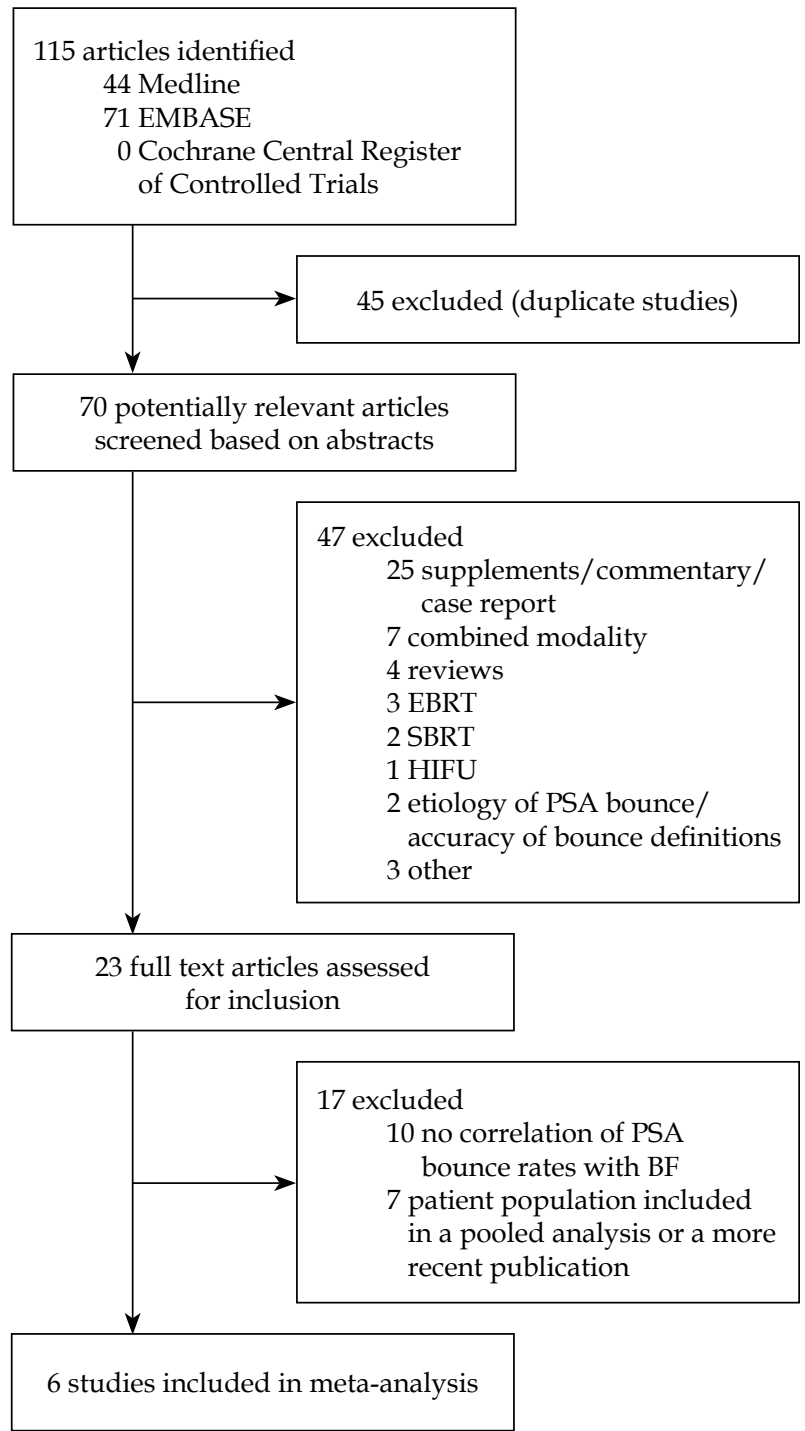

EBRT - external beam radiation therapy, SBRT - stereotactic body radiation therapy, HIFU - high-intensity focused ultrasound, PSA - prostate-specific antigen, BF-biochemical failure

Fig. 1. Selection of studies 


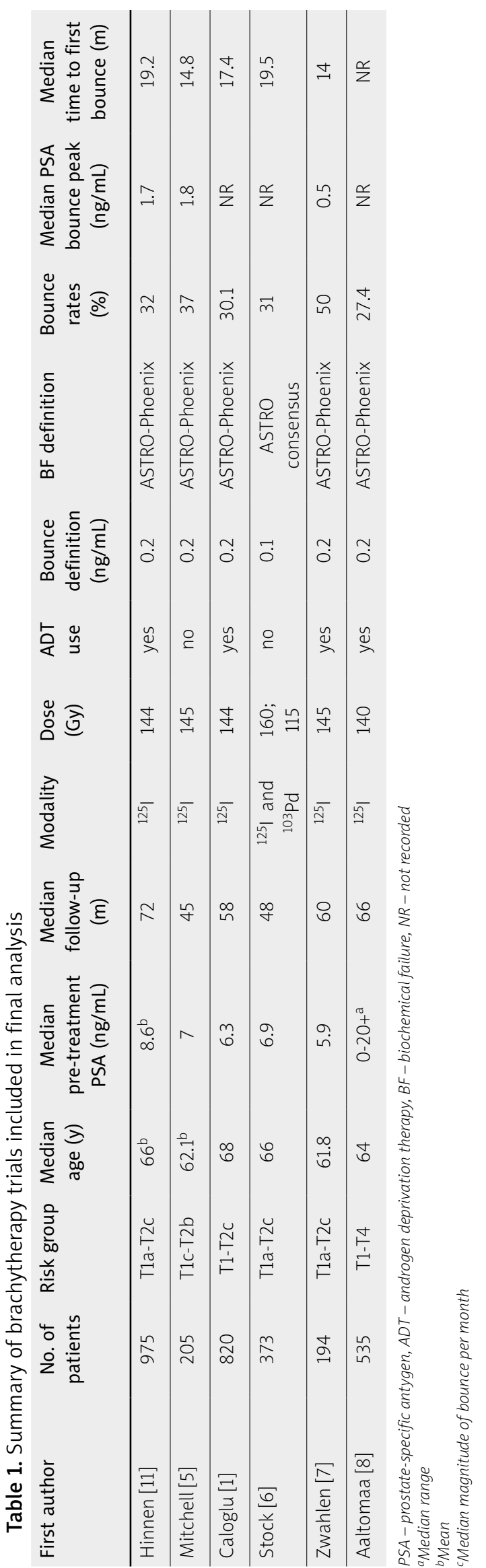

\section{Prognostic significance of PSA bounce following brachytherapy}

There were a total of 3011 patients treated with brachytherapy $\left({ }^{125} \mathrm{I}\right.$ or ${ }^{103} \mathrm{Pd}$ ) as a monotherapy in the six studies included in the final analysis. In five out of six reports, the ASTRO-Phoenix definition $(\geq 2 \mathrm{ng} / \mathrm{mL}$ above PSA nadir) was used for biochemical failure and a rise of $0.2 \mathrm{ng} /$ $\mathrm{mL}$ above a nadir defined a PSA bounce. One study used a rise of $\geq 0.1 \mathrm{ng} / \mathrm{mL}$ for the bounce threshold and the ASTRO consensus definition for biochemical failure [6]. Median follow-up ranged from 45 to 72 months, with a median time to first bounce between 17.4 and 19.5 months. PSA bounce rates were recorded in $27.4-50 \%$ of patients. There was evidence of heterogeneity between study results (Cochrane's $Q=10.25, p=0.086$ ). Random effects meta-analysis revealed that experiencing a PSA bounce after brachytherapy was associated with a statistically significant decrease in the risk of biochemical failure (HR $=0.42,95 \%$ CI: $0.30-0.59 ; p<0.001)$. Meta-analysis results are shown in Figure 2. No evidence of publication bias was detected using the Egger test $(p=0.947)$.

\section{Discussion}

Although the PSA bounce phenomenon has been welldescribed for years, its potential implication on biochemical control remains highly controversial. Select studies have shown improved rates of biochemical control in patients experiencing PSA bounce after brachytherapy alone [1], while other investigators have shown an increased incidence of biochemical recurrence in bouncers $[4,15]$. Additionally, certain studies have failed to confirm PSA bounce as a prognostic indicator [3,9]. Thus, we performed an up-to-date systematic review and meta-analy-

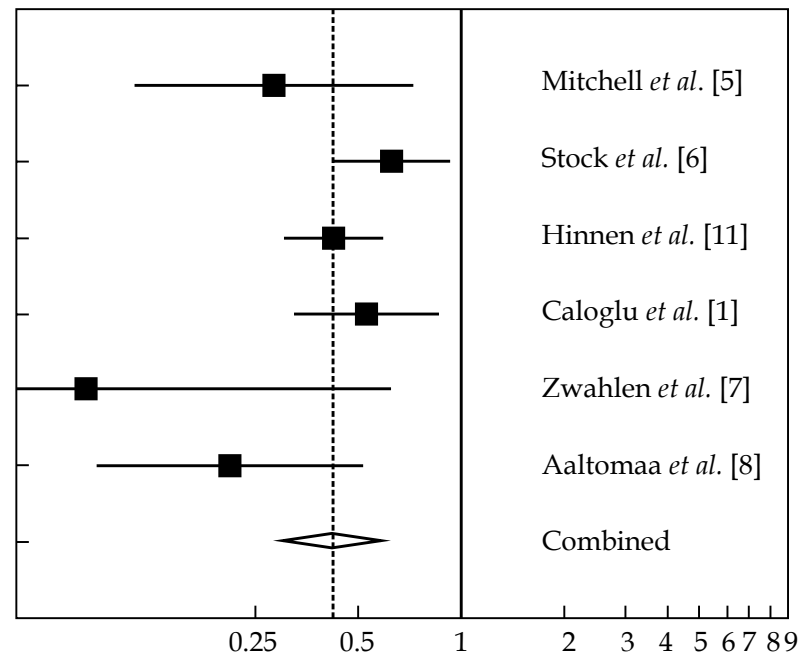

Hazard ratio for biochemical failure after PSA bounce

PSA - prostate-specific antigen

The hazard ratios of biochemical recurrence after brachytherapy were calculated using a random-effects model (HR $=0.42,95 \%$ CI: 0.30 $0.59, p<0.001]$. The diamond indicates the summary hazard ratio

Fig. 2. Forest plot of brachytherapy trials 
sis to determine if a post-radiation PSA bounce impacts biochemical control in prostate cancer patients treated with single modality therapy.

Our meta-analysis revealed that PSA bounce predicted for improved biochemical control following brachytherapy. The final analysis included six studies totalling 3011 patients treated with ${ }^{125} \mathrm{I}$ or ${ }^{103} \mathrm{Pd}$ as a monotherapy, and showed a statistically significant decrease in recurrence rates in patients meeting the threshold for PSA bounce. In all but one study, bounce was defined as a rise of $\geq 0.2 \mathrm{ng} / \mathrm{mL}$ above a nadir and the ASTRO-Phoenix criteria was used to define biochemical recurrence. Stock et al. defined bounce as a rise of $\geq 0.1 \mathrm{ng} / \mathrm{mL}$ above PSA nadir and used the ASTRO consensus definition for biochemical failure [6]. Had this study been excluded from the final analysis for consistency, the above findings would have remained the same (data not shown). Our findings are consistent with a recent publication that analyzed the clinicopathological and post-dosimetric parameters predictors of PSA bounce. In that report, no patient with PSA bounce after low-dose rate brachytherapy experienced biochemical recurrence, while 10/150 patients that did not meet the criteria for bounce were diagnosed with biochemical recurrence [16].

Interestingly, a large multi-institutional study by Horwitz et al., which pooled 4839 patients from nine institutions treated with external beam radiation therapy alone showed the opposite result, reporting biochemical control rates of $58 \%$ versus $72 \%$ at 10 years for bouncers and non-bouncers, respectively [4]. The contrasting implications of PSA bounce following EBRT found in that study compared to the conclusions that we report following brachytherapy have not been previously described. Some have theorized that PSA bounce is a positive prognostic factor because it signifies lethal en-mass cellular death and a favourable physiologic response to radiation therapy [17]. This phenomenon may be more likely after brachytherapy, where focal areas of the prostate and stroma are exposed to ablative radiotherapy doses that promote apoptotic cell death [18]. A second hypothesis is that the heterogeneous dose distribution of brachytherapy may promote late radionecrosis within the prostate. The resulting release of tumor-specific antigens into the bloodstream may stimulate a directed immune response, similar to the intensive lymphocytic infiltration into brain tumors seen after treatment with Gamma Knife radiosurgery [19]. Fractionated EBRT, on the other hand, is unlikely to be associated with these effects, which manifests clinically as lower rates of bounce, earlier time to bounce, and shorter duration of bounce [6] such that a PSA rise after therapy may represent true tumor regeneration.

While bounce rates are less frequent following EBRT, discrepancy exists when comparing bounce frequency following treatment with LDR versus HDR brachytherapy. According to a report from Pinkawa et al., PSA bounce rates were significantly higher following LDR brachytherapy compared to HDR brachytherapy ( $42 \%$ vs. $23 \%$, respectively). Bounces $>1 \mathrm{ng} / \mathrm{mL}$ were also more frequent after LDR than HDR brachytherapy [20]. The authors suggest that local inflammatory effects may play a major role for the predominant induction of bounces after LDR brachytherapy - even in contrast to HDR brachytherapy. Conversely, a more recent study reported bounce rates as high as $43 \%$ following HDR brachytherapy [21]. Perhaps these findings can be explained by examining the similarities and differences between these two techniques, including the ease of administration, side effect profile, dosimetry, and radiobiological implications [22]. Clearly, additional studies are necessary to reconcile these observations.

Regardless of the treatment modality, several patient characteristics that may predict for a post-radiation PSA bounce have been described, including risk group stratification, pretreatment PSA, Gleason score, PSA nadir, the use of androgen ablation, and dose to the prostate $[2,3,6,10]$. Despite dose escalation with EBRT, the recommended ${ }^{125}$ I monotherapy prescription dose for a prostate implant is 144 Gy - nearly twice what can be achieved with EBRT. Perhaps it is simply the higher dose to the prostate that can account for the increase in PSA bounce rates with brachytherapy. This brachytherapy prescription, however, has been recently called into question, and thus higher dose prescriptions for these implants may be on the horizon, potentially leading toward more patients experiencing a bounce [23].

Nevertheless, the clinical factor that appears most consistently to predict for PSA bounce after EBRT $[4,24]$ or brachytherapy $[3,5-8,11]$ is younger age. Increased sexually activity in this patient population has been proposed as one possible explanation for this observation $[2,10]$. Alternatively, a delayed apoptotic event may simply be more common in younger individuals [2]. Caloglu et al. also discussed that younger patients may have more androgen production and more reactive epithelial cells, which may increase the incidence of the bounce phenomenon $[1,6]$. Similarly, Akyol et al. described a direct relationship between rising testosterone levels and the occurrence of a PSA bounce, thereby suggesting that younger patients with higher testosterone levels may experience higher rates of PSA bounce [9]. As seen in Table 1, the brachytherapy series typically include younger men when compared to the EBRT literature, which may explain why most brachytherapy studies do a report a higher bounce rate than those using the EBRT technique. In addition to age, a lower threshold value used to meet the criteria for a bounce after brachytherapy may contribute as well since a PSA rise of $\geq 0.2 \mathrm{ng} / \mathrm{mL}$ to signal a bounce after brachytherapy, and $\geq 0.4 \mathrm{ng} / \mathrm{mL}$ following EBRT appear to be the most widely accepted definitions in the literature $[2,4,6,11,15]$.

We acknowledge that a potential limitation of our study is the use of different definitions for PSA bounce and biochemical failure for one of the brachytherapy papers included in our final analysis [6]. Some prior studies have confirmed the rates of bounce and biochemical control will be affected by which criteria are chosen $[1,6,7]$. Nevertheless, no study was excluded in our final analysis based on definitions alone, and when multiple definitions for bounce and biochemical recurrence were included in one study, the aforementioned definitions were chosen whenever possible. While a single definition of PSA bounce would be more appealing, the optimal characteri- 
zation for this phenomenon remains undefined. The same report also included patients treated with ${ }^{103} \mathrm{Pd}$ as the source, while all others treated patients with ${ }^{125} \mathrm{I}[6]$. However, those patients only accounted for $1.2 \%$ of our study population. Additionally, as stated above, 4 patients in one study received EBRT plus brachytherapy. This report was included in the final analysis as it only represented $4 / 3011(0.001 \%)$ of our study population. Furthermore, when this study was excluded from the meta-analysis, our findings remained the same (data not shown). Lastly, we could not assess the impact of bounce on other endpoints, such as distant metastases or overall survival, because these relationships are rarely reported in existing papers. Interestingly, Hinnen et al. found that bounce after prostate brachytherapy was related improved disease-specific survival and overall survival [11]. While understanding how PSA bounce affects development of distant metastases and overall survival would be informative, identifying its impact on biochemical progression-free survival is useful since patients are frequently salvaged with ADT, which can be associated with decreased quality of life [25].

\section{Conclusions}

Significance discordance currently exists in the literature whether PSA bounce has prognostic significance. Because this phenomenon occurs in many as $50 \%$ after brachytherapy [7], understanding its potential clinical relevance may be important when counselling patients regarding their future cancer care. Our meta-analysis showed that PSA bounce is a positive prognostic factor after brachytherapy and predicts for increased rates of biochemical control. To our knowledge, this is the first meta-analysis investigating this issue.

\section{Disclosure}

Authors report no conflict of interest.

\section{References}

1. Caloglu M, Ciezki JP, Reddy CA et al. PSA bounce and biochemical failure after brachytherapy for prostate cancer: a study of 820 patients with a minimum of 3 years of follow-up. Int J Radiat Oncol Biol Phys 2011; 80: 735-741.

2. Patel C, Elshaikh MA, Angermeier K et al. PSA bounce predicts early success in patients with permanent iodine-125 prostate implant. Urology 2004; 63: 110-113.

3. Crook J, Gillan C, Yeung I et al. PSA kinetics and PSA bounce following permanent seed prostate brachytherapy. Int J Radiat Oncol Biol Phys 2007; 69: 426-433.

4. Horwitz EM, Levy LB, Thames HD et al. Biochemical and clinical significance of the posttreatment prostate-specific antigen bounce for prostate cancer patients treated with external beam radiation therapy alone: a multiinstitutional pooled analysis. Cancer 2006; 107: 1496-1502.

5. Mitchell DM, Swindell R, Elliott T et al. Analysis of prostate-specific antigen bounce after $\mathrm{I}(125)$ permanent seed implant for localised prostate cancer. Radiother Oncol 2008; 88: 102-107.

6. Stock RG, Stone NN, Cesaretti JA. Prostate-specific antigen bounce after prostate seed implantation for localized prostate cancer: descriptions and implications. Int J Radiat Oncol Biol Phys 2003; 56: 448-453.
7. Zwahlen DR, Smith R, Andrianopoulos N et al. Prostate-specific antigen bounce after permanent iodine- 125 prostate brachytherapy - an Australian analysis. Int J Radiat Oncol Biol Phys 2011; 79: 179-187.

8. Aaltomaa SH, Kataja VV, Räty A et al. Does the outcome of prostate cancer patients with large prostates differ from small prostate size in permanent seed, low dose-rate brachytherapy? Scand J Urol Nephrol 2011; 45: 339-345.

9. Akyol F, Ozyigit G, Selek U et al. PSA bouncing after short term androgen deprivation and 3D-conformal radiotherapy for localized prostate adenocarcinoma and the relationship with the kinetics of testosterone. Eur Urol 2005; 48: 40-45.

10. Das $\mathrm{P}$, Chen $\mathrm{MH}$, Valentine $\mathrm{K}$ et al. Using the magnitude of PSA bounce after MRI-guided prostate brachytherapy to distinguish recurrence, benign precipitating factors, and idiopathic bounce. Int J Radiat Oncol Biol Phys 2002; 54: 698-702.

11. Hinnen KA, Monninkhof EM, Battermann JJ et al. Prostate specific antigen bounce is related to overall survival in prostate brachytherapy. Int J Radiat Oncol Biol Phys 2012; 82: 883-888.

12. Moher D, Liberati A, Tetzlaff J et al. Preferred reporting items for systematic reviews and meta-analyses: the PRISMA statement. Ann Intern Med 2009; 151: 264-269.

13. Parmar MK, Torri V, Stewart L. Extracting summary statistics to perform meta-analyses of the published literature for survival endpoints. Stat Med 1998; 17: 2815-2834.

14. Egger M, Davey Smith G, Schneider M et al. Bias in metaanalysis detected by a simple, graphical test. BMJ 1997; 315: 629-634.

15. Hanlon AL, Pinover WH, Horwitz EM et al. Patterns and fate of PSA bouncing following 3D-CRT. Int J Radiat Oncol Biol Phys 2001; 50: 845-849.

16. Tanaka N, Asakawa I, Fujimoto K et al. Minimal percentage of dose received by $90 \%$ of the urethra (\%UD90) is the most significant predictor of PSA bounce in patients who underwent low-dose-rate brachytherapy (LDR-brachytherapy) for prostate cancer. BMC Urol 2012; 12: 28.

17. Rosser CJ, Kuban DA, Levy LB et al. Prostate specific antigen bounce phenomenon after external beam radiation for clinically localized prostate cancer. J Urol 2002; 168: 2001-2005.

18. Fuks Z, Kolesnick R. Engaging the vascular component of the tumor response. Cancer Cell 2005; 8: 89-91.

19. Szeifert GT, Salmon I, Rorive $S$ et al. Does gamma knife surgery stimulate cellular immune response to metastatic brain tumors? A histopathological and immunohistochemical study. J Neurosurg 2005; 102 Suppl: 180-184.

20. Pinkawa M, Piroth MD, Holy R et al. Prostate-specific antigen kinetics following external-beam radiotherapy and temporary (Ir-192) or permanent (I-125) brachytherapy for prostate cancer. Radiother Oncol 2010; 96: 25-29.

21. Mehta NH, Kamrava M, Want PC et al. Prostate-specific antigen bounce after high-dose-rate monotherapy for prostate cancer. Int J Radiat Oncol Biol Phys 2013; 86: 729-733.

22. Skowronek J. Low-dose-rate or high-dose-rate brachytherapy in treatment of prostate cancer - between options. J Contemp Brachytherapy 2013; 5: 33-41.

23. Aronowitz JN, Rivard MJ. The phylogeny of permanent prostate brachytherapy. J Contemp Brachytherapy 2013; 5: 89-92.

24. Sheinbein C, Teh BS, Mai WY et al. Prostate-specific antigen bounce after intensity-modulated radiotherapy for prostate cancer. Urology 2010; 76: 728-733.

25. D'Amico AV, Chen MH, Renshaw AA et al. Androgen suppression and radiation vs radiation alone for prostate cancer: a randomized trial. JAMA 2008; 299: 289-295. 